\title{
Micro-scale assessment of the postoperative effect of anterior cruciate ligament reconstruction preclinical study using a 7.1T micro-magnetic resonance imaging
}

\author{
FANG CHAI $^{1,2}$, FANG WAN ${ }^{2}$, JIA JIANG ${ }^{2}$ and SHIYI CHEN ${ }^{2}$ \\ ${ }^{1}$ Department of Orthopedics, Zhejiang Provincial People's Hospital, People's Hospital of Hangzhou Medical College, \\ Hangzhou, Zhejiang 310003; ${ }^{2}$ Department of Sports Medicine and Arthroscopic Surgery, Huashan Hospital, \\ Sports Medicine Center, Fudan University, Shanghai 200040, P.R. China
}

Received October 2, 2017; Accepted March 1, 2018

DOI: $10.3892 /$ etm.2018.6080

\begin{abstract}
High-field micro-magnetic resonance imaging (MRI) scanning may provide additional information for quantitative analysis of graft bone healing processes, thus serving as a promising supplementary method in graft and bone healing evaluation following anterior cruciate ligament reconstruction (ACLR) surgery during preclinical studies. The present study included 12 New Zealand white rabbits that underwent ACLR with polyethylene terephthalate (PET) ligament. At 4, 8, and 16 weeks following surgery, 4 rabbits were euthanized and knee joint samples were harvested for a 7.1T micro-magnetic resonance imaging (MRI) scan. The graft bone tunnel diameter and signal noise ratio (SNR) at the region of interest (ROI) were measured. Hematoxylin-eosin staining was performed at each time point to verify the graft bone healing process in histology. The bone tunnel diameter at the graft tunnel interface decreased over time in both femoral and tibial parts. Notably, the tunnel size was smaller than the diameter of the drilling Kirschner wire that was used to observe the femoral part and proximal site of the tibial part at 16 weeks following surgery. SNR research demonstrated that both the femoral and tibial part PET ligaments selected in the ROI exhibited a marked increase in SNR from the initial 4-week results. The micro-MRI result was consistent with that of histological analysis. Micro-MRI scanning was applied in an animal model that underwent ACL reconstruction surgery with PET ligament, and it was determined that micro-MRI is promising in quantitatively
\end{abstract}

Correspondence to: Professor Shiyi Chen, Department of Sports Medicine and Arthroscopic Surgery, Huashan Hospital, Sports Medicine Center, Fudan University, 12 Middle Urumuqi Road, Shanghai 200040, P.R. China

E-mail: cshiyifudan@sina.com

Key words: anterior cruciate ligament, micro-magnetic resonance imaging, treatment effect assessment, signal noise ratio observing graft bone healing processes directly with a focus on graft tunnel distances and SNRs.

\section{Introduction}

The anterior cruciate ligament (ACL) is an important stabilizing structure of the knee, and ACL injury is listed as one of the most common injuries in sports medicine; $>120,000$ ACL reconstruction (ACLR) surgeries are reportedly performed annually in the United States alone (1). Early rehabilitation and return to sports following an operation are considered core objectives following an ACLR. To reach these targets, graft and bone healing is crucial in the postoperative course; therefore, various studies have been conducted under the scope of graft and bone healing processes (2-4).

In clinical studies, the graft and bone healing condition is primarily evaluated indirectly via physical examination for ligament stability (e.g. Lachman test or pivot-shift test (5) and return to sports capability (e.g. single-leg hop test, and isokinetic evaluation (6), subjective functional scoring scales (e.g. International Knee Documentation Committee (IKDC) Knee Ligament Evaluation Form (7) and a personal questionnaire regarding patients' return to sports, activity frequency and condition. Radiographic assessments are typically performed in the form of bilateral weight-bearing $\mathrm{X}$-ray scans based on the IKDC knee arthritis grading system (8). Recent studies have utilized a magnetic resonance imaging (MRI) scan to evaluate graft maturity; this approach may illustrate graft and bone healing conditions at each time point more clearly (9-11). For animal studies, histological examination is thought to be the most suitable method in the evaluation of graft bone healing processes. Specimens are typically harvested, fixed, embedded, sectioned and prepared for multiple staining protocols to evaluate different components, and graft and bone healing conditions are revealed clearly in slides under microscopy. However, a number of limitations are also present with histological examination modalities, as histology studies analyze graft and bone healing in segments, focusing more on the regional healing condition and failing to elaborate on the entire graft bone healing result. 

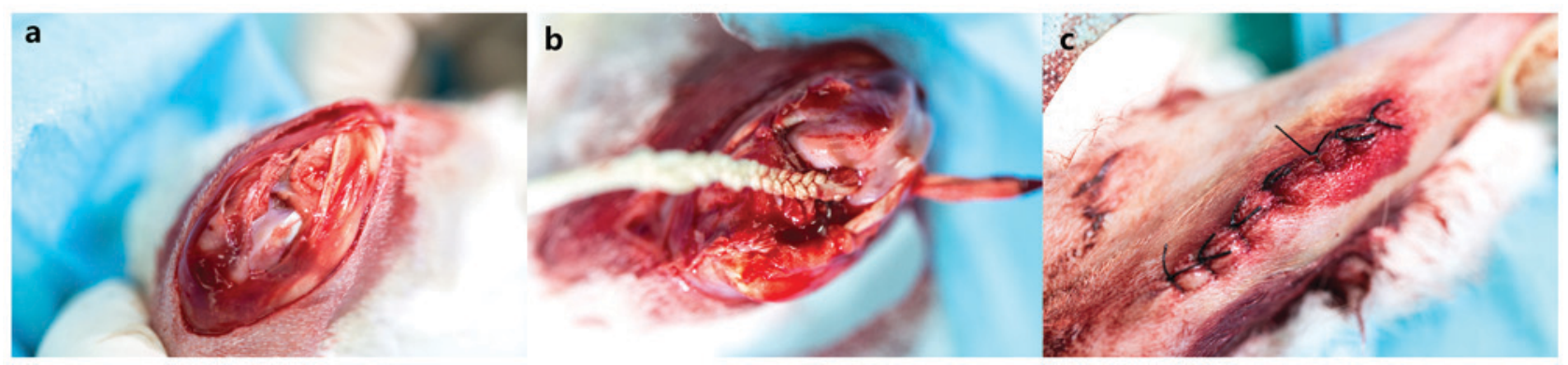

Figure 1. Representative images of the animal surgery procedure. (a) Medial patellar retinaculum release and exposure of knee joint. (b) Polyethylene terephthalate ligament fed through the bone tunnel. (c) Wound following suture in layers.

Micro-MRI has previously been introduced in preclinical studies in orthopedics research $(12,13)$. Due to its superiority in high spatial resolution, high soft tissue contrast and visualizing tendons, ligaments, cartilage, menisci and other non-osseous tissues directly, micro-MRI with a high field coil has been applied in a variety of osteoporosis, osteoarthritis and cartilage lesion animal studies $(14,15)$. Disease progression and cartilage derogation have been identified in animal models, including mouse, rat, rabbit and dog models (14-16). In the study of ACL, however, few studies have been performed with micro-MRI scanning as a possible method for evaluating graft bone healing processes. This may be associated with difficulties in accessing devices or lack of previous experience.

In the present study, high-field micro-MRI was performed on animal models that underwent ACLR surgery at different time points following surgery to assess progressive changes in tunnel diameter and MRI signal noise ratio in graft and bone structures. It was hypothesized that high-field micro-MRI scanning may provide additional information on graft bone healing processes, thus serving as a promising supplementary method in graft and bone healing evaluations following ACLR surgery during preclinical studies.

\section{Materials and methods}

Animal ACLR model. The present animal study was reviewed and approved by the Animal Experiment Ethics Committee of the College of Pharmacy, Fudan University (Shanghai, China). A total of 12 skeletally mature male New Zealand white rabbits (age, 1 year; weight, $3.4 \pm 0.4 \mathrm{~kg}$ ) were provided by the Animal Efficacy Evaluation Center, College of Pharmacy, Fudan University (Shanghai, China). Rabbits were housed at $20^{\circ} \mathrm{C}$ with a 40-60\% humidity under a 16/8 h light/dark cycle. Animals also received ad libitum access to food and water. Following 2 weeks of quarantine, ACLR surgeries were performed on the rabbits' right legs. Briefly, the rabbits were fixed on the surgery table in a supine position following general anesthesia with intravenous administration of $3 \%$ pentobarbital $(30 \mathrm{mg} / \mathrm{kg}$ body weight; Shanghai Wokai; Sinopharm Chemical Reagent Co., Ltd. Shanghai, China). A medial parapatellar incision was made, and the native ACL was exposed and transected. Joint laxity was confirmed by a Lachman test following removing the native ACL. A 3.0-mm diameter Kirschner wire was used to drill the bone tunnels from the ACL footprints and remnants towards the femoral and tibial insertion sites of the ACL. The polyethylene terephthalate (PET) ligament fabricated prior to surgery was fed through the tunnel with the help of a PDS II wire (Ethicon, Inc., Cincinnati, OH, USA). The femoral and tibial ends of the ligament were fixed with the periosteum adjacent soft tissue. Following graft transplantation, consecutive cycling loads were performed 20 times to ensure the ligaments were functional. Then, the wounds were sutured in layers (Fig. 1). Postoperatively, rabbits were returned to their cages and allowed free cage activity without immobilization. Intramuscular prophylactic antibiotic injections $(800,000 \mathrm{IU}$ penicillin) and wound cleaning were performed immediately following the operation and once daily for 3 consecutive days.

Rabbits were euthanized via administration of 3\% pentobarbital $(100 \mathrm{mg} / \mathrm{kg})$ in the auricular vein at 4,8 and 16 weeks following surgery and the femur-graft-tibia complex specimens ( $\mathrm{n}=4$ at each time point) were harvested immediately from the rabbits following sacrifice. Soft tissues around the complex were well preserved.

Micro-MRI scan and data analysis. In all rabbit specimens, MRI examinations were performed with a 7.1T Biospec 70/20R MRI magnet (Bruker AXS GmbH, Karlsruhe, Germany). An automatic shimming process was performed at the beginning of each scan to homogenize the magnetic field. Specimens were placed supine on the animal bed with a $38-\mathrm{mm}$ transmit-receive cylindrical birdcage radio frequency coil fixed to it. A two-dimensional T2-TurboRare sequence (repetition time, 3,500 ms; echo time, $26 \mathrm{~ms}$; flip angle, $90^{\circ}$; field of view, $16 \times 16 \mathrm{~mm}$; slice thickness, $0.35 \mathrm{~mm}$; gap between slices, $0 \mathrm{~mm}$; acquisition resolution, 256x256 matrix size) was used in the scanning.

The PET ligament and bone tunnel interface distance were measured in axial position images, and the distance was identified as the largest diameter from the center of the ligament fiber to the edge of the bone tunnel. The tunnel diameters at the proximal, middle and distal sites in the bone tunnel were measured simultaneously, both in the femoral and tibial tunnels (Fig. 2).

The signal noise ratio (SNR) is a measure of signal strength in a certain area relative to background noise. SNR analyses were performed using ImageJ 1.51e (National Institutes of Health, Bethesda, MD, USA). Briefly, the range of interest (ROI) was set based on the oblique sagittal plane in which the PET ligament graft and bone tunnels were mostly linear and parallel to each other in the longitudinal direction. The PET ligament in the femoral bone tunnel was highlighted in a linear region with a width of $10 \mathrm{~mm}$ set on the boundary of the 


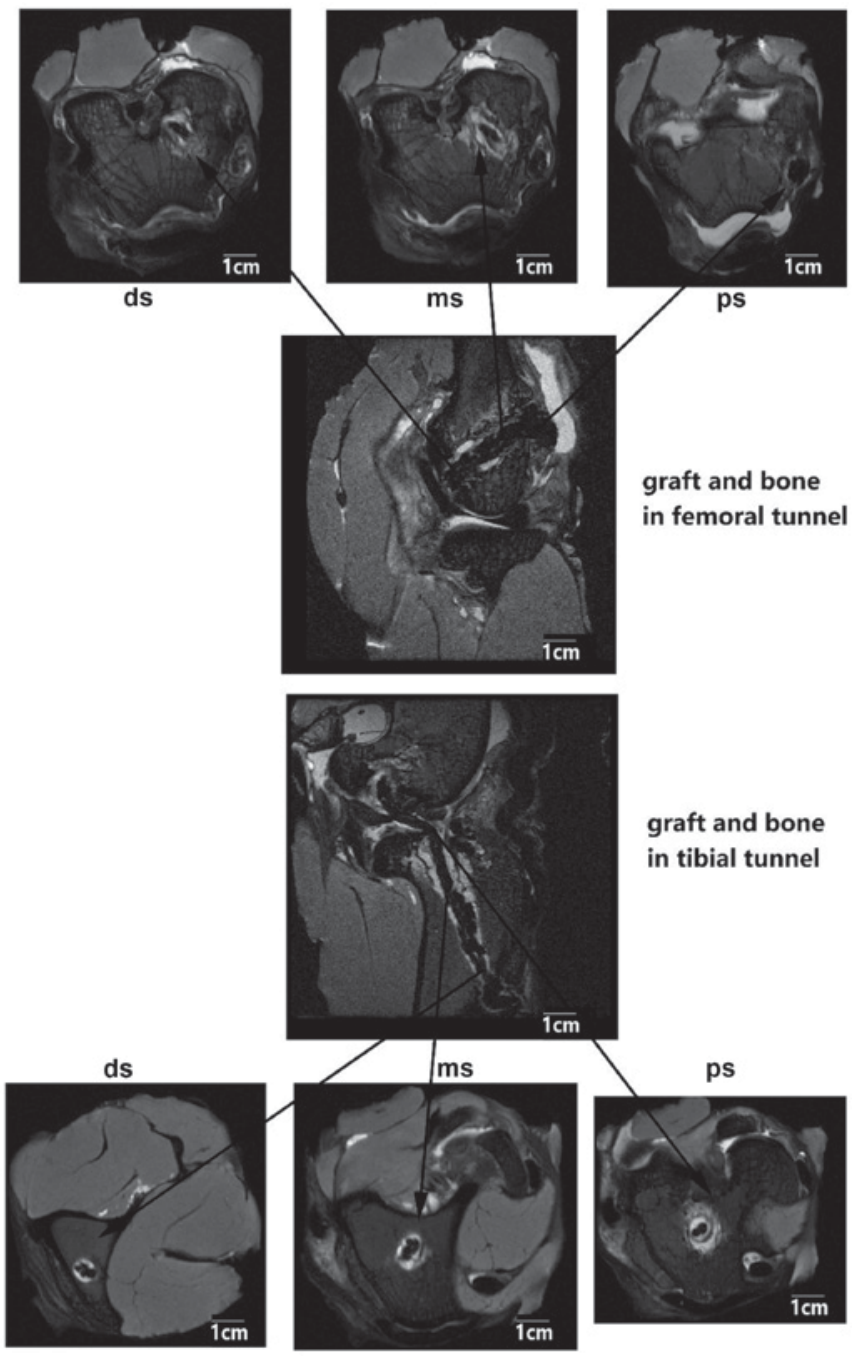

Figure 2. Micro-magnetic resonance imaging analysis of the graft transplanted tunnel diameter in the ps, ms and ds of the femoral and tibial parts. Ps, proximal site; ms, middle site; ds, distal site.

joint surface side parallel to Blumensaat's line. The PET ligament in the tibia bone tunnel was highlighted in a linear region with a width of $10 \mathrm{~mm}$ set on the boundary of the joint surface side parallel to the joint surface at the opening site of the bone tunnel. The signal intensity of the background was measured at $1 \mathrm{~cm}$ anterior to the tibia tuberosity. The SNR was calculated as signal intensity in the ROI divided by signal intensity in the background area (Fig. 3) (17). Two sports medicine surgeons who were blinded to the sample information at the measurement moment were requested to provide an analysis, and the mean values were regarded as final results.

Histological examination. Bone-graft complexes were harvested from each group at 4,8 and 16 weeks $(n=4$ at each time point) following micro-MRI and fixed in $10 \%$ formalin for $48 \mathrm{~h}$ at $10^{\circ} \mathrm{C}$ immediately prior to histological examination. Specimens prepared for undecalcified bone slicing were embedded in resin and sectioned with a sliding microtome (SM2500; Leica Microsystems GmbH, Wetzlar, Germany) perpendicular to the longitudinal axis of the femur and tibia tunnel at a thickness of $5 \mu \mathrm{m}$. The sections were stained at $20 \mathrm{kC}$ for $6 \mathrm{~h}$ with hematoxylin-eosin to evaluate the graft, host

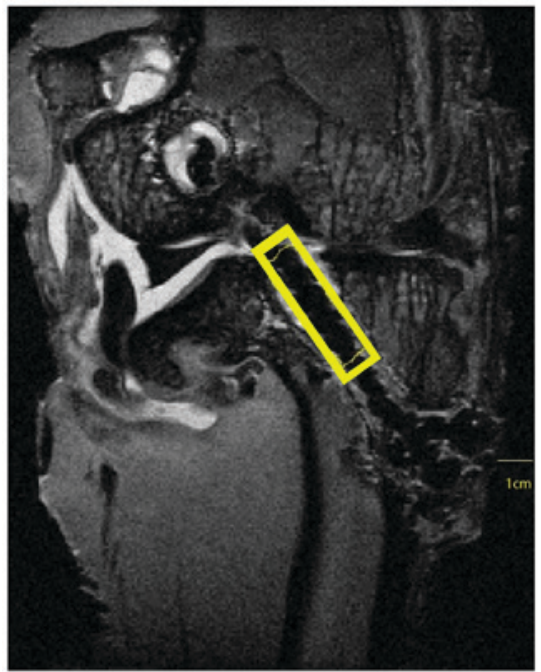

Figure 3. Representative image of signal noise ratio analysis region selection The highlighted region covering the polyethylene terephthalate ligament was chosen as the region of interest in which the signal intensity was acquired. The straight line anterior to the tibia tuberosity acts as a scale bar and as an indicator of a $1 \mathrm{~cm}$ distance from the knee joint to where the background noise data was collected.

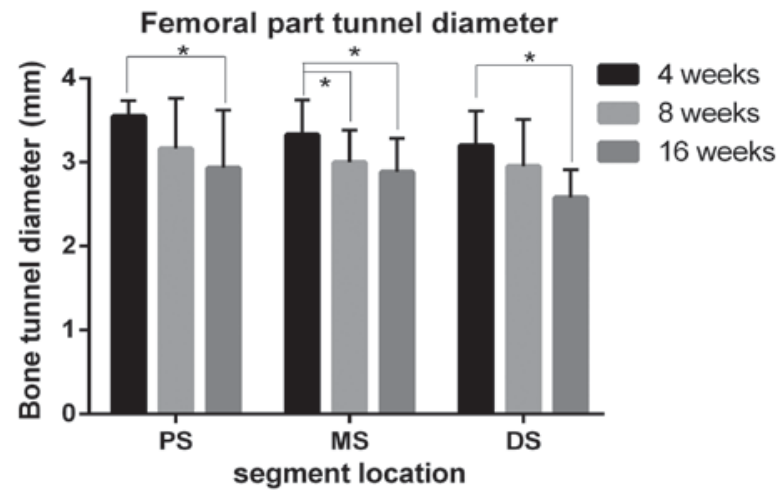

Figure 4. Graft bone tunnel diameter measured in the femoral part tunnel at 4, 8 and 16 weeks following surgery. Data are presented as the mean + standard deviation $(\mathrm{n}=4)$. ${ }^{*} \mathrm{P}<0.05$. PS, proximal site; MS, middle site; DS, distal site.

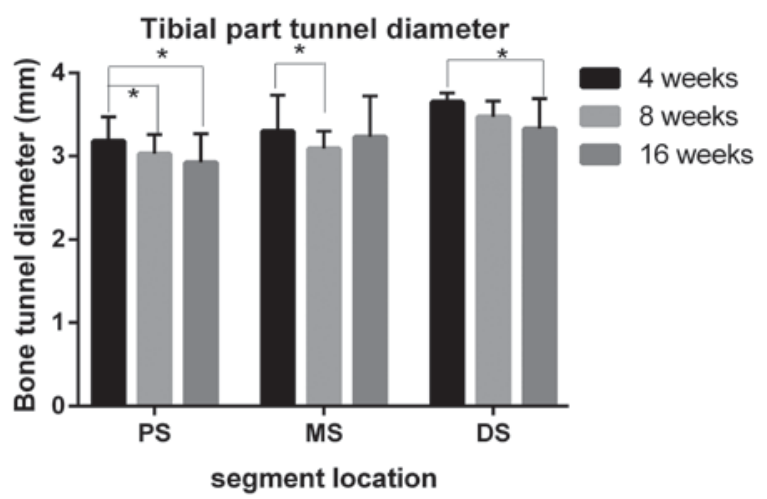

Figure 5. Graft bone tunnel diameter measured in the tibial part tunnel at 4, 8, and 16 weeks following surgery. Data are presented as the mean + standard deviation $(n=4)$. ${ }^{*} \mathrm{P}<0.05$. PS, proximal site; MS, middle site; DS, distal site.

bone and the interface between them. Images were visualized via inverted microscopy (IX71SBF2; Olympus Corporation, 

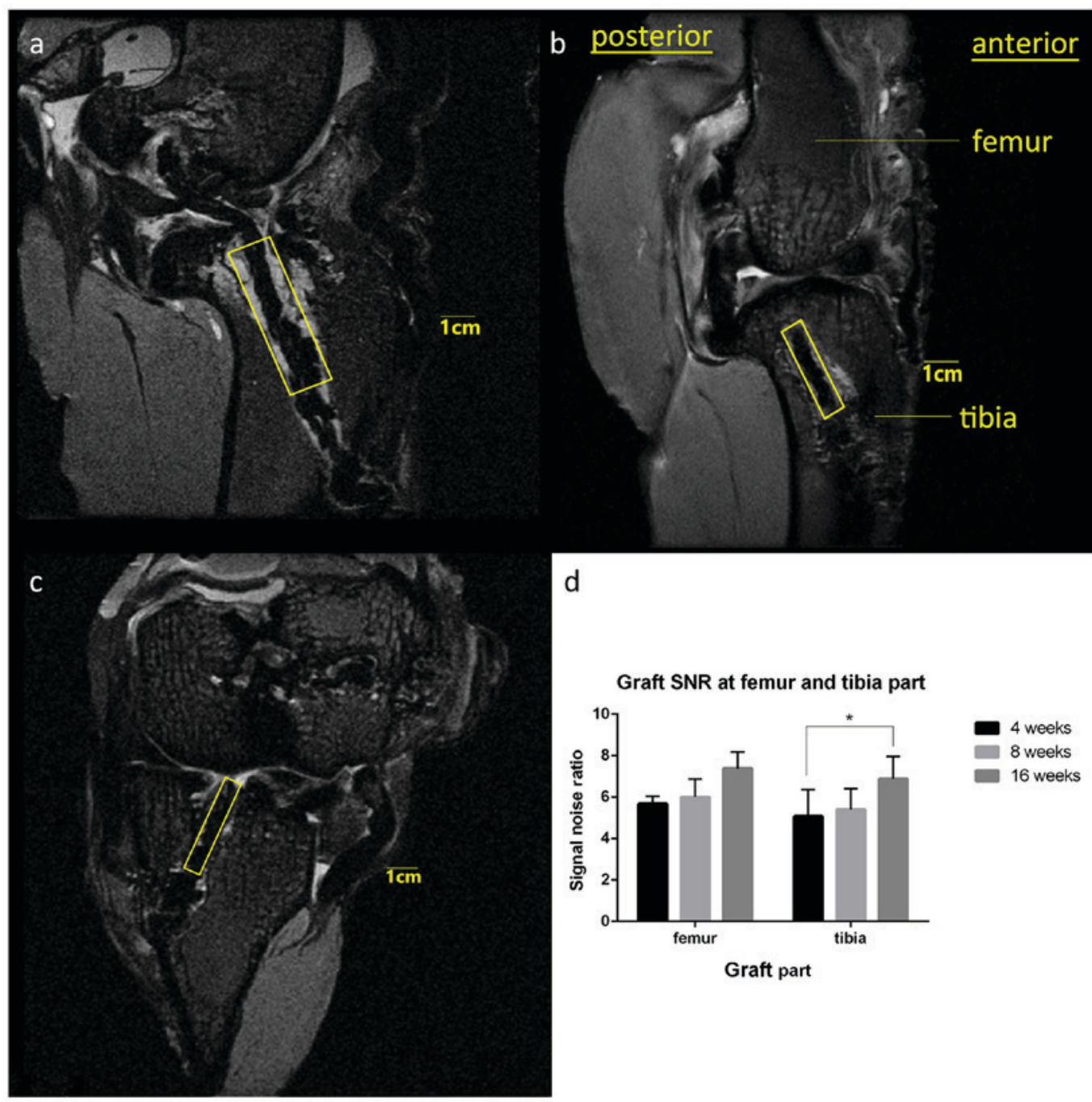

$\mathrm{d}$

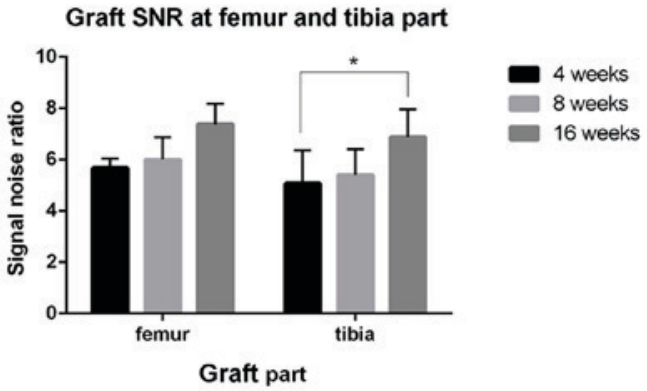

Figure 6. Polyethylene terephthalate ligament graft in the femoral and tibial parts in the range of interest (highlighted area) at (a) 4, (b) 8 , and (c) 16 weeks following surgery. (d) Quantitative analysis of SNR. Data are presented as the mean + standard deviation $(\mathrm{n}=4)$. ${ }^{\text {}} \mathrm{P}<0.05$. SNR, signal noise ratio.

Tokyo, Japan) and captured with DP72 Manager (Olympus Corporation).

Statistical analysis. Statistical analysis was performed using SPSS 22.0 (IBM Corp., Armonk, NY, USA). Data are presented as the mean \pm standard deviation. One-way analysis of variance with Fisher's Least Significant Difference was used to analyze the mean diameter between the graft and bone tunnel in the femoral and tibial tunnels, and the mean SNR at the femur and tibia. $\mathrm{P}<0.05$ was considered to indicate a statistically significant difference.

\section{Results}

Bone tunnel diameter measurements. The bone tunnel diameter at the graft tunnel interface site was largest at 4 weeks following surgery (femoral part, proximal site $=3.55 \pm 0.18 \mathrm{~mm}$, middle site $=3.33 \pm 0.41 \mathrm{~mm}$ and distal site $=3.20 \pm 0.41 \mathrm{~mm}$; Fig. 4; tibial part, proximal site $=3.15 \pm 0.41 \mathrm{~mm}$, middle site $=3.68 \pm 0.43 \mathrm{~mm}$, and distal site: $3.80 \pm 0.19 \mathrm{~mm}$; Fig. 5). Compared with samples at 4 weeks, the size was decreased in the samples collected at 8 and 16 weeks following surgery. At 8 weeks, the tunnel diameter at the middle site in the femoral part and at the proximal site in the tibial part showed significant shrinkage when compared with the results at the same site at 4 weeks $(\mathrm{P}<0.05)$. At 16 weeks following surgery, all 6 measuring sites, except the tibial medial site, in the tibial tunnel reported significantly decreased tunnel diameters in comparison with the 4-week results $(\mathrm{P}<0.05)$. It is worthwhile to note that in certain measuring sites (proximal, middle and distal site of the femur part and proximal site of the tibia part; diameters $=2.93 \pm 0.69,2.88 \pm 0.40,2.58 \pm 0.33$ and $2.92 \pm 0.35 \mathrm{~mm}$, respectively), the tunnel diameter was smaller than the diameter of the Kirschner wire used in tunnel drilling at 16 weeks following surgery.

SNR analysis. SNR analysis revealed that both the femoral and tibial PET ligaments selected in the ROI exhibited a marked increase in SNR from the initial 4-week results (femoral at 4 weeks, 5.68 \pm 0.36 ; tibial at 4 weeks, $5.08 \pm 1.28$ ). However, statistical significance was only detected in the comparison between 16 and 4 weeks in the SNR value in the tibia $(\mathrm{P}=0.0464$; Fig 6).

Evaluation of graft bone interfaces. As presented in Fig. 7, the interface between host bone and the PET ligament graft was 

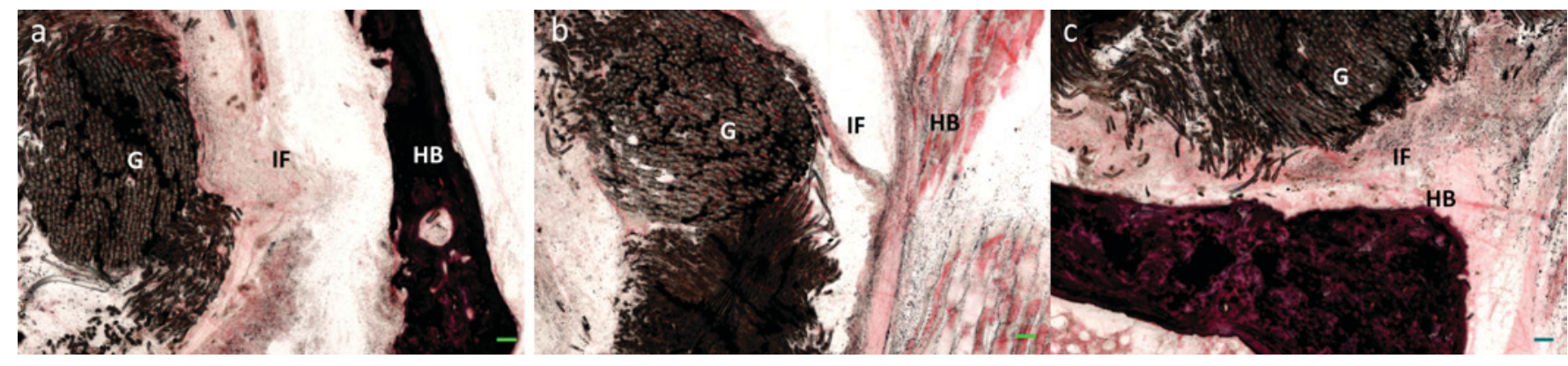

Figure 7. Representative histologic images of the graft bone interfaces at (a) 4, (b) 8, and (c) 16 weeks following surgery (hematoxylin-eosin staining; magnification, x200). Scale bar $=100 \mu \mathrm{m}$. IF, interface; HB, host bone; G, artificial ligament graft.

notable at 4 weeks following surgery. At 8 weeks following animal surgery, the gap interface had shrank but remained visible via hematoxylin-eosin staining. At 16 weeks, the PET graft was surrounded with ingrowth bony tissue and the interface was markedly smaller than that observed at 4 weeks.

\section{Discussion}

The graft and bone healing processes following surgery heavily affect the therapeutic effects as well as patients' abilities to return to sports, which has always been emphasized in sports medicine practice (1). In the hope of accelerating the ligamentation process, various methods have been applied in preclinical studies $(18,19)$. To analyse the association between those enhancement solutions and graft bone healing processes, daily observation, gross observation, anatomic study, biomechanics test, histology, and medical imaging were included in the present study. New Zealand Rabbits are frequently utilized in animal experimental research. The size and morphological similarity to human knee joint made the animal an appropriate choice in ACL animal studies. ACLR surgery has also become a common choice for the treatment of ACL injury.

Daily observation, though not performed in the present study due to resource limitation, may be carried out prior to and following the ACLR surgery. Items listed in the daily observation include, but are not limited to, the animal's weight, hair color, wound healing condition, knee joint flexion and extension angle regarding range of motion and active activity level (19). These indicators partially reflect the animal's quality of life following surgery and, to a certain extent, the graft functions in daily movement. However, difficulties in quantitative analysis with the majority of these indicators impede further progress of indicator analysis (20). The most frequently used assessment system of ACLR may be the adjusted Oswestry Arthroscopy Score (OAS) on a macroscopic assessment. Items listed on the evaluation form include levels at the insertion site with the surrounding cartilage, integration at the insertion site with the surrounding tissue, stiffness of the tendon graft, appearance of articular surface and color of the articular surface, with a total score of 10 (21). Gross evaluation provides investigators with an initial semi-quantitative analysis of the samples and set a rational foundation for further study. Cheng et al (18) recently used the OAS in the assessment of fresh specimens harvested from ACLR rabbit models. As an assessment scale output results in ordinal data, some evaluation bias is inevitable due to results at boundaries between the two classes. Anatomic study following the sample harvest provides an opportunity to further understand general anatomical structure following graft transplantation and its association with structures adjacent to it $(22,23)$. Like the daily observation, those studies failed to offer quantitative data for assessment. Molecular biology study refers to the testing of certain biomedical markers associated with ligament integration processes based on the serum or synovium extracted during or following caging (24-26). By conducting molecular biology study, researchers may determine whether certain enhancement methods induce a concentration rise in certain growth factors and proteins that have been renowned for their cartilage or bone incorporation capacity. Functional biological networks, however, should not be neglected, as it is believed that signals and factors function through networks instead of a simple downstream signal or factor. Thus, a confounding bias should be noted. Biomechanical testing has been emphasized in recent years in ACLR research such as testing dynamic stress loads and stiffness with certain fixing devices including a Biopuls prosthetic limb fixture test and a three-point bending/breaking device $(27,28)$. The boundedness of biomechanical testing is a result of the improper design and use of a fixation device at the beginning of a test. Certain vehicles, including prosthetic limb fixture testing devices may fail to stretch the knee joint in a functional way, resulting in misleading data (27,28). Histology study is crucial in ACLR preclinical study. Slides stained with hematoxylin and eosin, toluidine blue, Masson's trichrome stain, and safranin $\mathrm{O}$ help researchers observe grafts, newly born bone, graft-bone interface and types of collagen specifically and clearly (29-32). Examination via histology also has some disadvantages. The mechanical and biological properties of the whole ACL structure are evaluated using localized sections. Furthermore, tissue wastage during slicing hinders continuous observation of the graft bone complex.

Imaging study methods used previously in ACLR preclinical study primarily involve X-ray and micro computed tomography (micro-CT) scans. The former is beneficial in knee joint arthritis detection based on the Kellgren and Lawrence system and IKDC scale, offering second-hand ACL graft healing information (29). Micro-CT scan has been used in the study of bone architecture and mineral density during preclinical investigations of osteoporosis in several animal models $(31,32)$. High contrast quality enables a non-invasive method in evaluating changes in bone stereology and bone trabecular structure following ACLR $(30,31)$. However, the ligament grafted in the 
surgery is not markedly developed under screening as ligament grafts are typically made with materials that are low density and are close to the air background.

Micro-MRI is a novel study solution tool in ACLR preclinical studies. With the use of small high-efficiency coils and high field imagery, micro-MRI is superior in soft tissue imaging. In osteoarthritis animal models, non-invasive quantitative high field micro-MRI is more specific for quantitatively detecting menisci and cartilage lesions at different stages when compared with the results used with common 3 T MRI devices $(11,16,30)$.

With the use of micro-MRI, the present study demonstrated that graft tunnel diameters decreased over time following ACLR surgery. The preliminary result was rationalized as a micro-bone fracture due to drilling in the tunnel formation, which was unavoidable, and the following callus formation and remodeling are natural courses for fracture healing, resulting in decreases in the diameters. On further examination, however, at 16 weeks following surgery it was observed that the diameter of the whole femoral and partial tibial parts were $<3 \mathrm{~mm}$, which was the diameter of the Kirschner wire used in drilling. A difference of $0.4 \mathrm{~mm}$ between the observed and estimated diameter at the distal site in the femoral part may be attributed to bone incorporation from the surrounding area, as reported recently in the use of micro-CT scanning on ACLR in animal models $(2,30)$. Inward growth decreased the graft bone interference distance in micro-scale assessment. An alternative explanation is required for the variance in diameters among proximal, middle and distal sites in both femoral and tibial parts. This is primarily due to the technique used in drilling the tunnel. The tunnel was initially drilled from the native ACL remnants within the knee joint towards femoral and tibial insertion sites. To feed the PET ligament through the tunnel, extra drilling was performed to expand the tunnel size at the femoral and tibial sites of the tunnel. SNR analysis provided another perspective in evaluating graft conditions following ACLR. The graft used in ACLR was set to present a relatively fixed signal intensity. The rise in SNR between 4 and 16 weeks following surgery in both femoral and tibial parts of the PET ligament graft indicated potential tissue growth on the surface or inside the ligament graft, which is consistent with the aforementioned decreases in diameters. Based on the hematoxylin-eosin staining results, it was demonstrated that the results were consistent with micro-MRI findings at 4, 8 and 16 weeks. The interface decreased over time, which was in accordance with micro-MRI findings. These findings suggest that micro-MRI is an effective tool and possible supplement to the histology, biomechanical study, gross evaluation, and micro-CT scans in the ACLR preclinical research field.

However, there are several limitations to the present study. First, a small sample size of 12 New Zealand white rabbits was investigated. Further studies with larger sample amounts are required for better analysis of micro-MRI. Second, due to the limitation of the coil size, in vivo micro-MRI scanning, which is accessible for rats and mice in preclinical study, was not available in the current study, as reported above. With in vivo observation, a single knee joint may be continuously observed at different time points. Despite the short interval between euthanasia, harvesting and micro-MRI scanning, the in vitro micro-MRI scan may not fully reflect the true structural circumstance inside the knee joint. A more suitable MRI coil may solve this problem. Third, a further limitation is the imaging of the graft bone complex samples with micro-MRI. The T2-Turborare Sequence used in the present study may detect the ligament and cartilage lesion more easily than T1 sequences, but specific scan protocols, such as T2 mapping, fat saturated gradient echo, and 3D gradient echo, may be more feasible in subsequent research on ACLR preclinical studies $(31,32)$. Furthermore, CT scanning or Micro-CT scanning would be helpful in tunnel diameter evaluation in our future study.

The present study applied micro-MRI scans in an animal model that underwent ACLR surgery with a PET ligament, and determined that micro-MRI is promising in quantitatively observing graft bone healing processes directly, with a focus on graft tunnel distances and the SNR.

\section{Acknowledgements}

Not applicable.

\section{Funding}

The present study was supported by grants from the National 863 Hi-Tech Project (grant no. 2015AA033703), the National Natural Science Foundation of China (grant nos. 81271958, 81572108 and 81370052) and the Specialized Research Fund for the Doctoral Programme of Higher Education (grant no. 20120071110067).

\section{Availability of data and materials}

The datasets used and/or analyzed during the current study are available from the corresponding author on reasonable request.

\section{Authors' contributions}

FC and FW performed the experiment and wrote the manuscript. JJ conceived the idea and designed the study with SC. In addition, $\mathrm{SC}$ reviewed and rewrote the manuscript.

\section{Ethics approval and consent to participate}

The present study was reviewed and approved by the Animal Experiment Ethics Committee of the College of Pharmacy, Fudan University (Shanghai, China).

\section{Consent for publication}

Not applicable.

\section{Competing interests}

The authors declare that they have no competing interests.

\section{References}

1. Mall NA, Chalmers PN, Moric M, Tanaka MJ, Cole BJ, Bach BR Jr and Paletta GA Jr: Incidence and trends of anterior cruciate ligament reconstruction in the United States. Am J Sports Med 42: 2363-2370, 2014. 
2. Dong S, Huangfu X, Xie G, Zhang Y, Shen P, Li X, Qi J and Zhao J: Decellularized versus Fresh-frozen allografts in anterior cruciate ligament reconstruction: An in vitro study in a rabbit model. Am J Sports Med 43: 1924-1934, 2015.

3. Kuang GM, Yau WP, Lu WW and Chiu KY: Use of a strontium-enriched calcium phosphate cement in accelerating the healing of soft-tissue tendon graft within the bone tunnel in a rabbit model of anterior cruciate ligament reconstruction. Bone Joint J 95-B: 923-928, 2013.

4. Matsumoto T, Kubo S, Sasaki K, Kawakami Y, Oka S, Sasaki H, Takayama K, Tei K, Matsushita T, Mifune Y, et al: Acceleration of tendon-bone healing of anterior cruciate ligament graft using autologous ruptured tissue. Am J Sports Med 40: 1296-1302, 2012.

5. Katz JW and Fingeroth RJ: The diagnostic accuracy of ruptures of the anterior cruciate ligament comparing the Lachman test, the anterior drawer sign, and the pivot shift test in acute and chronic knee injuries. Am J Sports Med 14: 88-91, 1986.

6. Undheim MB, Cosgrave C, King E, Strike S, Marshall B, Falvey É and Franklyn-Miller A: Isokinetic muscle strength and readiness to return to sport following anterior cruciate ligament reconstruction: Is there an association? A systematic review and a protocol recommendation. Br J Sports Med 49: 1305-1310, 2015.

7. Hefti F, Müller W, Jakob RP and Stäubli HU: Evaluation of knee ligament injuries with the IKDC form. Knee Surg Sports Traumatol Arthrosc 1: 226-234, 1993.

8. van Meer BL, Meuffels DE, van Eijsden WA, Verhaar JA, Bierma-Zeinstra SM and Reijman M: Which determinant predict tibiofemoral and patellofemoral osteoarthritis after anterior cruciate ligament injury? A systematic review. Br J Sports Med 49: 975-983, 2015

9. Li H, Tao H, Cho S, Chen S, Yao Z and Chen S: Difference in graft maturity of the reconstructed anterior cruciate ligament 2 years postoperatively: A comparison between autografts and allografts in young men using clinical and 3.0-T magnetic resonance imaging evaluation. Am J Sports Med 40: 1519-1526, 2012.

10. Packer JD, Bedi A, Fox AJ, Gasinu S, Imhauser CW, Stasiak M, Deng XH and Rodeo SA: Effect of immediate and delayed high-strain loading on tendon-to-bone healing after anterior cruciate ligament reconstruction. J Bone Joint Surg Am 96: 770-777, 2014.

11. Wachsmuth L, Lindhorst E, Wrubel S, Hadzhiyski H, HudelmaierM,EcksteinFandChrubasikS:Micro-morphometrical assessment of the effect of Harpagophytum procumbens extract on articular cartilage in rabbits with experimental osteoarthritis using magnetic resonance imaging. Phytother Res 25: 1133-1140, 2011.

12. Lan SM, Wu YN, Wu PC, Sun CK, Shieh DB and Lin RM: Advances in noninvasive functional imaging of bone. Acad Radiol 21: 281-301, 2014

13. Wehrli FW: Structural and functional assessment of trabecular and cortical bone by micro magnetic resonance imaging. J Magn Reson Imaging 25: 390-409, 2007.

14. Boulocher C, Chereul E, Langlois JB, Armenean M, Duclos ME, Viguier E, Roger T and Vignon E: Non-invasive in vivo quantification of the medial tibial cartilage thickness progression in an osteoarthritis rabbit model with quantitative 3D high resolution micro-MRI. Osteoarthritis Cartilage 15: 1378-1387, 2007.

15. Libicher M, Ivancic M, Hoffmann M and Wenz W: Early changes in experimental osteoarthritis using the Pond-Nuki dog model: Technical procedure and initial results of in vivo MR imaging. Eur Radiol 15: 390-394, 2005.

16. Bolbos R, Benoit-Cattin H, Langlois JB, Chomel A, Chereul E, Odet C, Pastoureau P, Janier M and Beuf O: Knee cartilage thickness measurements using MRI: A 4(1/2)-month longitudinal study in the meniscectomized guinea pig model of OA. Osteoarthritis Cartilage 15: 656-665, 2007.

17. Kanamura H, Arai Y, Hara K, Takahashi T, Ikoma K, Fujiwara H, Minami G, Terauchi R, Nakagawa S, Honjo K and Kubo T: Quantitative evaluation of revascularization at bone tunnels and grafts with contrast-enhanced magnetic resonance angiography after anterior cruciate ligament reconstruction. Int Orthop 40: $1531-1536,2016$
18. Cheng P, Han P, Zhao C, Zhang S, Wu H, Ni J, Hou P, Zhang Y, Liu J, Xu H, et al: High-purity magnesium interference screws promote fibrocartilaginous entheses regeneration in the anterior cruciate ligament reconstruction rabbit model via accumulation of BMP-2 and VEGF. Biomaterials 81: 14-26, 2016.

19. Ma R, Ju X, Deng XH and Rodeo SA: A novel small animal model of differential anterior cruciate ligament reconstruction graft strain. J Knee Surg 28: 489-495, 2015.

20. Kondo E, Yasuda K, Katsura T, Hayashi R, Kotani Y and Tohyama $\mathrm{H}$ : Biomechanical and histological evaluations of the doubled semitendinosus tendon autograft after anterior cruciate ligament reconstruction in sheep. Am J Sports Med 40: 315-324, 2012.

21. Goebel L, Orth P, Müller A, Zurakowski D, Bücker A, Cucchiarini M, Pape D and Madry H: Experimental scoring systems for macroscopic articular cartilage repair correlate with the MOCART score assessed by a high-field MRI at 9.4 T-comparative evaluation of five macroscopic scoring systems in a large animal cartilage defect model. Osteoarthritis Cartilage 20: 1046-1055, 2012

22. Lee JK, Lee S, Seong SC and Lee MC: Anatomy of the anterior cruciate ligament insertion sites: Comparison of plain radiography and three-dimensional computed tomographic imaging to anatomic dissection. Knee Surg Sports Traumatol Arthrosc 23 : 2297-2305, 2015.

23. Zhao L, Thambyah A and Broom ND: A multi-scale structural study of the porcine anterior cruciate ligament tibial enthesis. J Anat 224: 624-633, 2014.

24. Fu SC, Cheuk YC, Yung SH, Rolf CG and Chan KM: Systematic review of biological modulation of healing in anterior cruciate ligament reconstruction. Orthop J Sports Med 2: $2325967114526687,2014$.

25. Kuang GM, Yau WP, Lu WW and Chiu KY: Osteointegration of soft tissue grafts within the bone tunnels in anterior cruciate ligament reconstruction can be enhanced. Knee Surg Sports Traumatol Arthrose 18: 1038-1051, 2010.

26. Proffen BL, Sieker JT and Murray MM: Bio-enhanced repair of the anterior cruciate ligament. Arthroscopy 31: 990-997, 2015.

27. Vavken P, Fleming BC, Mastrangelo AN, Machan JT and Murray MM: Biomechanical outcomes after bioenhanced anterior cruciate ligament repair and anterior cruciate ligament reconstruction are equal in a porcine model. Arthroscopy 28: 672-680, 2012

28. Gille J, Gerlach U, Oheim R, Hintze T, Himpe B and Schultz AP: Functional outcome of septic arthritis after anterior cruciate ligament surgery. Int Orthop 39: 1195-1201, 2015.

29. Bi F, Shi Z, Liu A, Guo P and Yan S: Anterior cruciate ligament reconstruction in a rabbit model using silk-collagen scaffold and comparison with autograft. PLoS One 10: e0125900, 2015.

30. Wachsmuth L, Keiffer R, Juretschke HP, Raiss RX, Kimmig N and Lindhorst E: In vivo contrast-enhanced micro MR-imaging of experimental osteoarthritis in the rabbit knee joint at 7.1T1. Osteoarthritis Cartilage 11: 891-902, 2003.

31. Batiste DL, Kirkley A, Laverty S, Thain LM, Spouge AR and Holdsworth DW: Ex vivo characterization of articular cartilage and bone lesions in a rabbit ACL transection model of osteoarthritis using MRI and micro-CT. Osteoarthritis Cartilage 12: 986-996, 2004.

32. Chu CR, Williams AA, West RV, Qian Y, Fu FH, Do BH and Bruno S: Quantitative magnetic resonance imaging UTE-T2* mapping of cartilage and meniscus healing after anatomic anterior cruciate ligament reconstruction. Am J Sports Med 42: 1847-1856, 2014

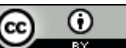

This work is licensed under a Creative Commons Attribution 4.0 International (CC BY 4.0) License. 